\title{
Interstitial pneumonitis secondary to long-term nitrofurantoin use with an improved clinical course secondary to corticosteroids
}

\author{
Baseer Quraishi BS, Jasmin Rahesh MBA, Mark Sigler MD
}

\section{INTRODUCTION}

Nitrofurantoin is an antibiotic that is frequently used for prophylaxis of recurrent urinary tract infections (UTIs). ${ }^{1}$ It can cause acute, subacute, or chronic lung injury. ${ }^{2}$ Acute lung injury consists of either a hypersensitivity reaction or eosinophilic pneumonia that can develop within days of use. Chronic injury consists of interstitial pneumonitis and fibrosis and is probably dose related., ${ }^{1,2}$ Nitrofurantoin should be discontinued if lung injury is suspected due to the possibility of irreversible injury. ${ }^{3}$ Many cases of nitrofurantoininduced lung injury resolve solely with drug discontinuation; Robinson was unable to establish a difference in outcomes in patients receiving corticosteroids for chronic lung injury. ${ }^{1,3-5}$ We present two cases of lung injury that had positive clinical response to systemic corticosteroid therapy along with discontinuation of nitrofurantoin.

\section{CASES}

\section{CASE 1}

A 68-year-old woman with a medical history significant for recurrent UTI and chronic hypoxemic respiratory failure presented to the hospital with a 15-day history of progressive shortness of breath and a witnessed syncopal episode. She was hypoxemic with an $\mathrm{O}_{2}$ saturation in the $40 \mathrm{~s}$ and was placed on high flow oxygen. She also had dry cough and fatigue. Vital signs were notable for a respiratory rate of 25 breaths per minute. Rhonchi were heard in both lung bases.

Two years prior to presentation, she was placed on daily nitrofurantoin for recurrent UTIs. One year

Corresponding author: Jasmin Rahesh

Contact Information: Jasmin.Rahesh@ttuhsc.edu DOI: 10.12746/swrccc.v10i42.937

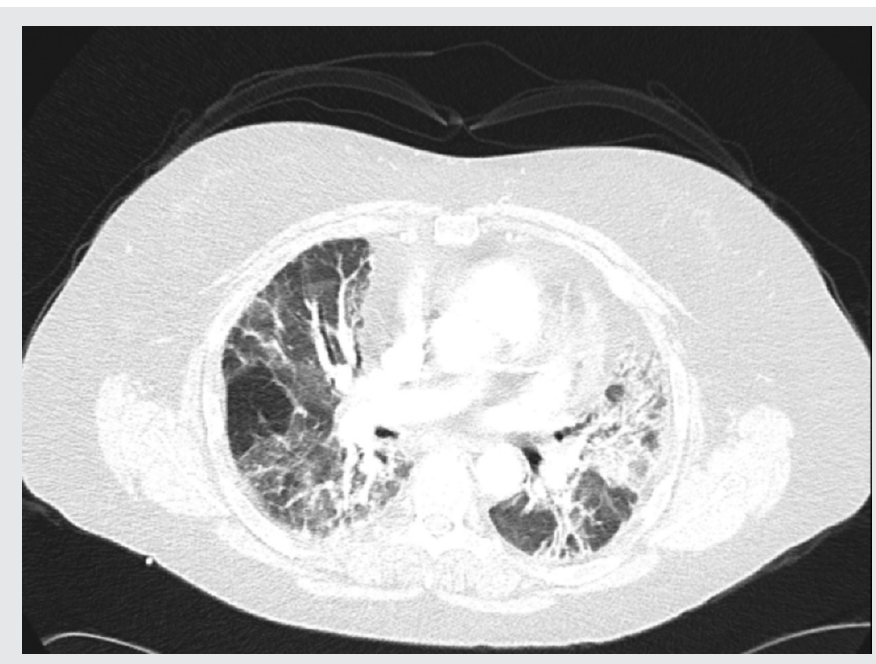

Figure 1. Chest computed tomography reveals significant fibrotic changes.

ago, she established care with a pulmonologist due to gradual onset shortness of breath with exertion and dry cough and was placed on $6 \mathrm{~L}$ of oxygen per minute. Surgical biopsy at that time suggested cryptogenic organizing pneumonia, and she was subsequently started on prednisone, azathioprine, and cyclophosphamide. The patient denied any history of smoking. Her WBC count was $9.1 \times 10^{9} / \mathrm{L}$ with $79.9 \%$ neutrophils and $3.7 \%$ eosinophils. A chest $x$-ray showed bilateral patchy opacities. Chest computed tomography (CT) showed bilateral ground glass opacities with extensive interstitial thickening (Figure 1).

Nitrofurantoin was discontinued, and the patient was started on IV methylprednisolone before switching to a 7-day course of $40 \mathrm{mg}$ oral prednisone that was subsequently reduced to $20 \mathrm{mg}$. Her cyclophosphamide and azathioprine were also held. At discharge, oxygen saturation was stable on $5 \mathrm{~L}$ per minute by nasal cannula with moderate exertional dyspnea that was improved in comparison to admission. She was scheduled for outpatient follow-up. 


\section{CASE 2}

A 77-year-old man presented to the pulmonary clinic at the request of his cardiologist for evaluation of dyspnea and cough. The patient had a 30-pack year smoking history but quit smoking 31 years ago. He denied use of any inhalers. The patient reported that he had a history of chronic UTIs treated with nitrofurantoin intermittently for the last $4-5$ years. He noticed the onset of dyspnea 6 weeks prior to presentation and reported increased dyspnea with exertion. At this time, it was suspected that this patient had nitrofurantoin-induced interstitial pneumonitis since nitrofurantoin was last prescribed 2 months prior to presentation for a 1-month course. His nitrofurantoin was stopped, and he was placed on $40 \mathrm{mg}$ of prednisone daily for 1 week, then $30 \mathrm{mg}$ daily for 1 week, and then $20 \mathrm{mg}$ daily for a week. A chest CT was ordered (Figure 2).

The week before the patient's next appointment he called reporting worsening shortness of breath when tapering off the prednisone. He was started on $20 \mathrm{mg}$ prednisone daily again. At follow-up he noted marked improvement in shortness of breath. Pulmonary function tests (PFTs) demonstrated FEV1/FVC 0.72, FEV1 1.77L

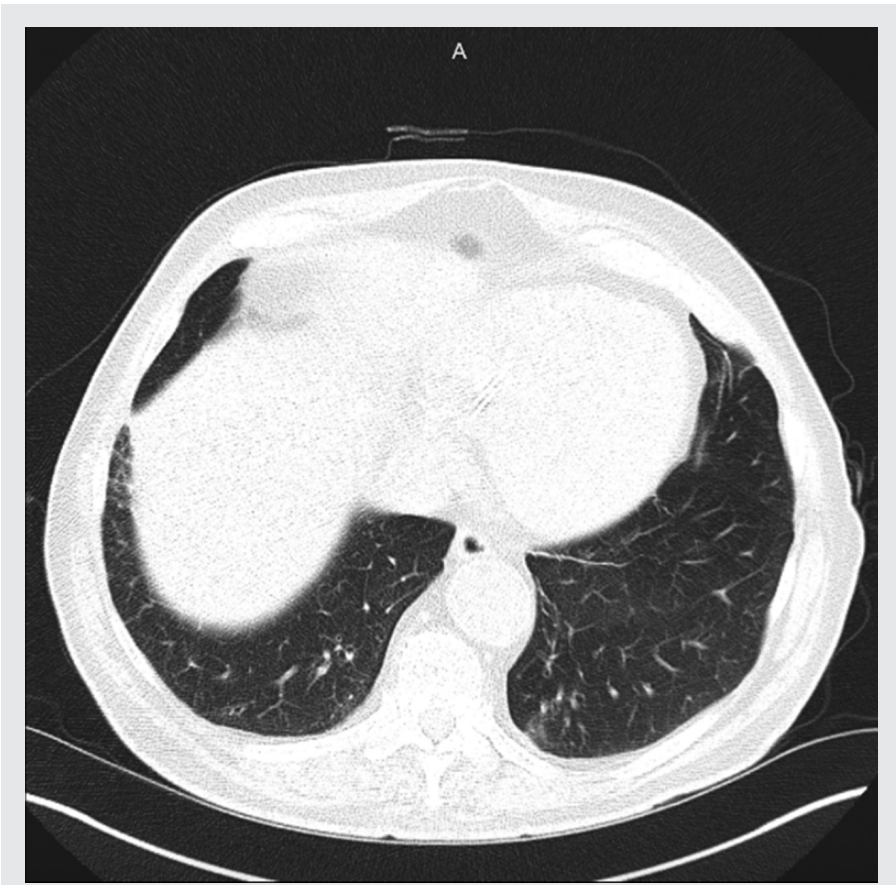

Figure 2. Chest computed tomography displays minimal fibrotic change.
(57\% predicted), TLC $79 \%$ predicted, and DLCO 98\% predicted. Another attempt at tapering prednisone was made with a plan to decrease the dose to $10 \mathrm{mg}$ daily.

At the patient's second 1-month follow-up, he reported increased shortness of breath with tapering of prednisone and had continued his $20 \mathrm{mg}$ daily dose. Another plan was made to taper the steroids to $15 \mathrm{mg}$ daily for 1 month and then $10 \mathrm{mg}$ daily until his next visit in 2 months. Repeat PFTs demonstrated improvement with a FEV1 $2.26 \mathrm{~L}$ (73\% predicted) and TLC 85\% predicted.

At the patient's third follow-up visit, he reported marked improvement and tapering of prednisone to $10 \mathrm{mg}$ daily with reports that some days he would administer the steroid every other day. A plan was made to taper his prednisone to $5 \mathrm{mg}$ daily every other day for 1 month with a follow-up visit with plans to have a non-contrast chest CT. At the patient's fourth follow-up visit he reported tapering of prednisone to $5 \mathrm{mg}$ every other day over the past month and no prednisone use for the past 2 weeks. The patient's prednisone was stopped at this visit.

\section{Discussion}

Nitrofurantoin is an antibiotic that is frequently used for treatment of uncomplicated UTIs and for prophylaxis of recurrent UTIs. ${ }^{1}$ A meta-analysis of its efficacy suggested that it is at least comparable to other antibiotics. ${ }^{5}$ Nitrofurantoin is generally a safe drug that can present with non-severe adverse effects, such as headache, nausea, vomiting, and diarrhea. ${ }^{5,6}$ In addition, more rare and serious reactions can occur. The most concerning side effects involve pulmonary toxicity that usually occurs with long-term use. This toxicity can be divided into acute, subacute, or chronic lung injury. ${ }^{7}$

Acute lung injury can present within days of administration of the drug with symptoms of cough, dyspnea, and fever and crackles on auscultation. ${ }^{7}$ Lab findings include eosinophilia, leukocytosis, and elevated inflammatory markers. Imaging findings include reticular changes and pleural effusions on chest $\mathrm{x}$-ray. ${ }^{7,9}$ Ground glass opacities can be seen on high resolution chest $\mathrm{CT} .{ }^{9}$ Biopsy specimens demonstrate interstitial inflammation and alveolar exudates. ${ }^{6}$

Chronic lung injury occurs with prolonged use and is seen more often in elderly women. ${ }^{10}$ In a review of 
921 cases by Holmberg and coauthors, the median onset of symptoms was after more than 12 months of use. ${ }^{7,8}$ Chronic lung injury presents with dry cough, dyspnea, and fatigue. Fever and eosinophilia usually do not occur with chronic injury.,12 Physical examination findings are non-specific and can include inspiratory crackles and reduced peripheral oxygen saturations. Both subacute and chronic lung injury can result in interstitial lung disease and sclerosis of pulmonary capillaries with findings on high-resolution computed tomography showing diffuse ground glass opacities, inter- and intralobular septal thickening of lower lobe predominance, bronchiectasis, and honeycombing. ${ }^{3,13}$

The most important treatment for lung injury secondary to long-term nitrofurantoin use is discontinuation of the drug, after which most patients usually recover within the next several weeks without any other interventions. ${ }^{8}$ Another treatment choice includes the addition of corticosteroids. However, there are no studies or data supporting corticosteroids as an effective treatment strategy in reducing the course of lung injury. This additional therapy is used when patients present with severe respiratory distress. ${ }^{11}$ We speculate that patient 2 presented in the acute phase of lung injury, with a more marked clinical response to systemic corticosteroid therapy. Although patient 1 demonstrated modest clinical and radiographic improvement with systemic steroid therapy, her prolonged course prior to presentation suggests that she may have progressed to chronic fibrotic lung disease, which may be less reversible with steroid therapy than acute lung injury.

Article citation: Quraishi B, Rahesh J, Sigler M. Interstitial pneumonitis secondary to long-term nitrofurantoin use with an improved clinical course secondary to corticosteroids. The Southwest Respiratory and Critical Care Chronicles 2022;10(42):28-30

From: Department of Internal Medicine, Texas Tech University Health Sciences Center, Amarillo, Texas

Submitted: $12 / 21 / 2021$

Accepted: $1 / 14 / 2022$

Conflicts of interest: none

This work is licensed under a Creative Commons Attribution-Share A like 4.0 International License.

\section{REFERENCES}

1. Chin AJ, Rashid S, Gharibeh TR, et al. Interstitial lung disease secondary to long-term nitrofurantoin Use. Am J Case Rep 2020;21:e920386. doi:10.12659/AJCR.920386

2. Lee B, Balavenkataraman A, Sanghavi D, et al. Recurrent nitrofurantoin-induced giant cell interstitial pneumonia: Case report and literature review. Respir Med Case Rep 2015; 14:49-52.

3. Syed H, Bachuwa G, Upadhaya S, et al. Nitrofurantoininduced interstitial pneumonitis: albeit rare, should not be missed. BMJ Case Rep. 2016;2016: bcr2015213967. doi:10. 1136/bcr-2015-213967

4. Robinson BW. Nitrofurantoin-induced interstitial pulmonary fibrosis. Presentation and outcome. Med J Aust 1983;1: 72-6.

5. Muller AE, Verhaegh EM, Harbarth S, et al. Nitrofurantoin's efficacy and safety as prophylaxis for urinary tract infections: a systematic review of the literature and meta-analysis of controlled trials. Clin Microbiol Infec 2017;23(6):355362.

6. Porreca A, D'Agostino D, Romagnoli D, et al. The clinical efficacy of nitrofurantoin for treating uncomplicated urinary tract infection in adults: a systematic review of randomized control trials. Urol Int 2021;105(7-8):531-540.

7. Sovijärvi AR, Lemola M, Stenius B, et al. Nitrofurantoinacute, subacute and chronic pulmonary reactions. Scand J Respir Dis 1977;58(1):41-50.

8. Holmberg L, Boman G, Böttiger LE, Eriksson B, Spross R, Wessling A. Adverse reactions to nitrofurantoin. Analysis of 921 reports. Am J Med. 1980;69(5):733-738. doi:10.1016/ 0002-9343(80)90443-X

9. Livanios K, Karampi ES, Sotiriou A, et al. Nitrofurantoininduced acute pulmonary toxicity. Respirol Case Rep 2016; 4(1):25-27.

10. Davis JW, Jones LS. Pleural effusion: An uncommon manifestation of nitrofurantoin-induced pulmonary injury. Respir Med Case Rep 2016;19:65-67.

11. Mendez JL, Nadrous HF, Hartman TE, et al. Chronic nitrofurantoin-induced lung disease. Mayo Clin Proc 2005;80(10): 1298-1302.

12. Batzlaff $C$, Koroscil M. Nitrofurantoin-induced pulmonary toxicity: always review the medication list. Cureus 2020; 12(8):e9807. Published 2020 Aug 17. doi:10.7759/cureus. 9807

13. Goemaere NN, Grijm K, van Hal PT, et al. Nitrofurantoininduced pulmonary fibrosis: a case report. J Med Case Rep 2008;2:169. Published2008May21.doi:10.1186/1752-19472-169 